Article

\title{
A Method for Direct Measurement of the First-Order Mass Moments of Human Body Segments
}

\author{
Yusaku Fujii ${ }^{1, *}$, Kazuhito Shimada ${ }^{2}$, Koichi Maru ${ }^{1}$, Junichi Ozawa ${ }^{1}$ and Rong-Sheng Lu ${ }^{3}$ \\ 1 Department of Electronic Engineering, Gunma University, 1-5-1 Tenjin-cho, Kiryu, Gunma, \\ 376-8515, Japan \\ 2 Space Medical Operations, Japan Aerospace Exploration Agency (JAXA) Tsukuba Space Center, \\ Tsukuba, 305-8505, Japan \\ 3 School of Instrument Science and Opto-electronic Engineering, Hefei University of Technology, \\ Hefei, Anhui, 230009, China \\ * Author to whom correspondence should be addressed; E-Mail: fujii@el.gunma-u.ac.jp; \\ Tel.: +81-277-30-1756; Fax: +81-277-30-1757.
}

Received: 7 July 2010; in revised form: 2 September 2010 / Accepted: 28 September 2010 / Published: 12 October 2010

\begin{abstract}
We propose a simple and direct method for measuring the first-order mass moment of a human body segment. With the proposed method, the first-order mass moment of the body segment can be directly measured by using only one precision scale and one digital camera. In the dummy mass experiment, the relative standard uncertainty of a single set of measurements of the first-order mass moment is estimated to be $1.7 \%$. The measured value will be useful as a reference for evaluating the uncertainty of the body segment inertial parameters (BSPs) estimated using an indirect method.
\end{abstract}

Keywords: body segment inertial parameter; center of mass; biomechanics

\section{Introduction}

The knowledge of body segment inertial parameters (BSPs) is required in the field of biomechanics. These parameters can be accurately estimated using dual x-ray absorptiometry (DXA) [1-3]. Although some DXA-based two-dimensional computation methods can be considered a direct measurement, the instrumentation for DXA is expensive and is not available to most researchers. In addition, the DXA- 
based three-dimensional BSP computation [4] is not a real direct measurement since it is necessary to assume mass densities to reconstruct a three-dimensional mass distribution. On the other hand, many simple models for estimating BSPs have also been proposed [5,6], and data derived from DXA are sometimes used as the standard.

In this paper, a simple and direct method for measuring the first-order mass moment, $\mathrm{M}^{(1)}=\mathrm{ML}$, is proposed, where $\mathrm{M}$ is the mass of a body segment and $\mathrm{L}$ is the length from the axis of rotation to the position of the center of mass $(\mathrm{CoM})$ of the segment.

In the proposed method, the first-order mass moment of a human body segment $\mathrm{M}^{(1)}$ is obtained from the two positions of the CoM of the entire body, in which the body segment under test has two different rotation angles. Only one precision scale and one digital camera are used in the experiment.

The first-order mass moment obtained with the proposed method can be used to evaluate the validity of the values of the BSPs obtained from other non-direct measurement methods. The moment value is also used for the posture error analysis in measuring human body masses in spacecraft with Space Scale [7-9].

\section{Methods}

Figure 1 shows the principle of the top-down view of the proposed method. The center of mass of the entire body can be expressed using the center of mass of the body segment $S_{1}$ and that of the rest of the body $\mathrm{S}_{2}$ as follows:

$$
\mathrm{CoMM}=\mathrm{CoM}_{1} \mathrm{M}_{1}+\mathrm{CoM}_{2} \mathrm{M}_{2}
$$

where $\mathrm{CoM}, \mathrm{CoM}_{1}$, and $\mathrm{CoM}_{2}$ are the center of mass of the entire body, of the body segment $\mathrm{S}_{1}$, and of the rest of the body $S_{2}$, respectively. $M, M_{1}$, and $M_{2}$ are the mass of the entire body, of the body segment $\mathrm{S}_{1}$, and of the rest of the body $\mathrm{S}_{2}$, respectively.

Figure 1. Principle of the method (top-down view).

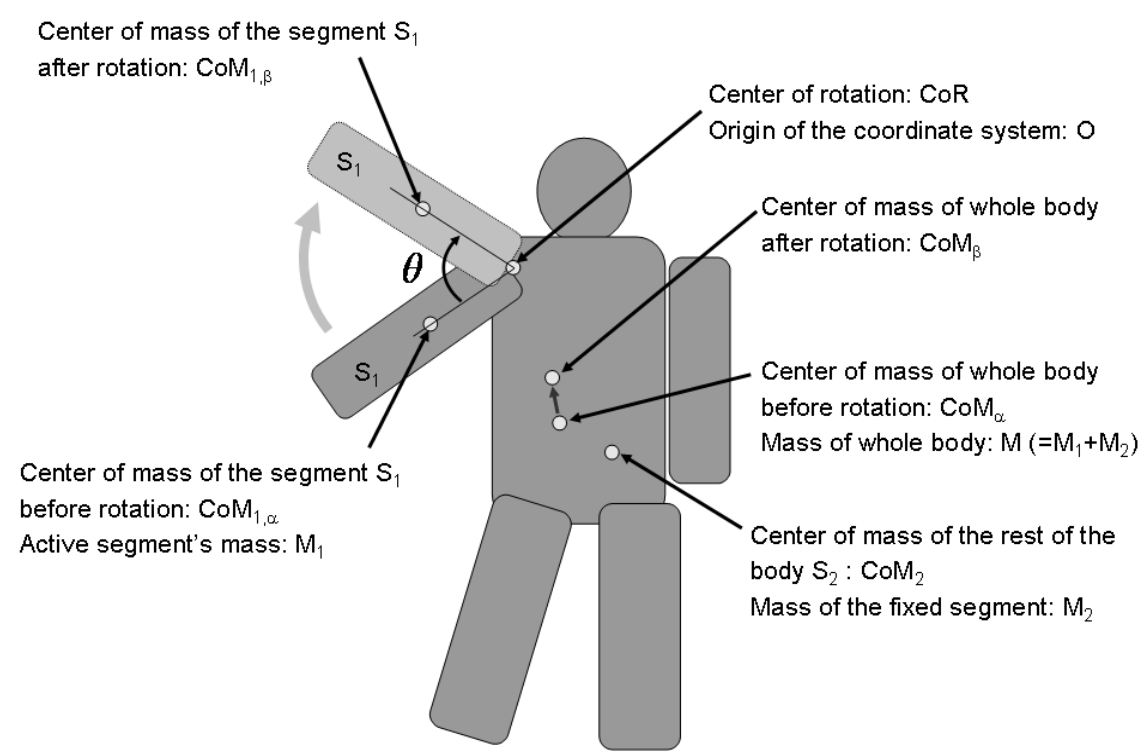


Equation (1) can be expressed as follows when the body segment under test $\mathrm{S}_{1}$ has two different rotation angles:

$$
\begin{aligned}
& \operatorname{CoM}_{\alpha} M=\operatorname{CoM}_{1 \alpha} M_{1}+\operatorname{CoM}_{2} M_{2} \\
& \operatorname{CoM}_{\beta} M=\operatorname{CoM}_{1, \beta} M_{1}+\operatorname{CoM}_{2} M_{2}
\end{aligned}
$$

where suffixes $\alpha$ and $\beta$ correspond to the two different states of the body obtained when the segment under test $\mathrm{S}_{1}$ has two different rotation angles. When the rotation occurs in the horizontal plane, $\mathrm{CoM}_{1, \beta}$ can be expressed using the rotation matrix $\mathrm{R}(\theta)$ and $\mathrm{CoM}_{1, \alpha}$ as follows:

$$
\begin{aligned}
& \mathrm{CoM}_{1, \beta}=(\mathrm{R}(\theta))\left(\mathrm{CoM}_{1, \alpha}\right) \\
& \mathrm{R}(\theta)=\left(\begin{array}{ll}
\cos \theta & -\sin \theta \\
\sin \theta & \cos \theta
\end{array}\right)
\end{aligned}
$$

where $\theta$ is the rotation angle of the segment under test $S_{1}$ from the initial state (expressed using suffix $\alpha$ ) to the final state (expressed using suffix $\beta$ ). On the basis of Equations (2) and (3), $\mathrm{CoM}_{2} \mathrm{M}_{2}$ can be eliminated as follows:

$$
\begin{aligned}
& \left(\mathrm{CoM}_{\alpha}-\operatorname{CoM}_{\beta}\right) \mathrm{M}=\left(\mathrm{CoM}_{1, \alpha}-\mathrm{CoM}_{1, \beta}\right) M_{1} \\
& \left(\mathrm{CoM}_{\alpha}-\operatorname{CoM}_{\beta}\right) \mathrm{M}=(\mathrm{I}-\mathrm{R}(\theta))\left(\mathrm{CoM}_{1, \alpha}\right) \mathrm{M}
\end{aligned}
$$

where I is the identity matrix. Then, the first-order mass moment $\mathrm{M}^{(1)}{ }_{1}$ of the segment under test $\mathrm{S}_{1}$ can be calculated using the rotation matrix $\mathrm{R}(\theta)^{-1}$; the center of mass of the entire body before and after the rotation of segment $\mathrm{S}_{1}$, i.e., $\mathrm{CoM}_{\alpha}$ and $\mathrm{CoM}_{\beta}$; and the mass of the entire body $\mathrm{M}$ as follows:

$$
\mathrm{M}_{1}^{(1)}=\left|\mathrm{CoM}_{1, \alpha}\right| \mathrm{M}_{1}=\left|(\mathrm{I}-\mathrm{R}(\theta))^{-1}\left(\mathrm{CoM}_{\alpha}-\mathrm{CoM}_{\beta}\right)\right| \mathrm{M}
$$

The values of $\mathrm{CoM}$ at the two different states are measured using a common technique [10,11]. Figure 2 shows the setup for measuring the CoM of an entire body.

The center of rotation of segment $\mathrm{S}_{1}, \mathrm{CoR}$, is set as the origin of the coordinate system, $\mathrm{O}$. The entire body is placed on a horizontal board, which is supported by the following three points: Support Point-1, Support Point-2, and Support Point-3. The weights applied at these three points are measured using a precision scale (capacity $3100 \mathrm{~g}$, resolution: $0.1 \mathrm{~g}$, precision: $1.5 \mathrm{~g}$, model: GX-30KR, manufacturer: A and D Co., Ltd. (Japan)) one by one, while the attitude of the body is fixed. During the measurement, the three support points are supported by the scale or the height adjustment blocks whose total height is equal to that of the scale. CoM is calculated as follows:

$$
\mathrm{CoM}=\left(\mathrm{P}_{\mathrm{P} 1} \mathrm{M}_{\mathrm{P} 1}+\mathrm{P}_{\mathrm{P} 2} \mathrm{M}_{\mathrm{P} 2}+\mathrm{P}_{\mathrm{P} 3} \mathrm{M}_{\mathrm{P} 3}\right) /\left(\mathrm{M}_{\mathrm{P} 1}+\mathrm{M}_{\mathrm{P} 2}+\mathrm{M}_{\mathrm{P} 3}\right)
$$

where $\mathrm{P}_{\mathrm{P} 1}, \mathrm{P}_{\mathrm{P} 2}$, and $\mathrm{P}_{\mathrm{P} 3}$ are the horizontal positions of Support Point-1, Support Point-2, and Support Point-3, respectively. $\mathrm{M}_{\mathrm{P} 1}, \mathrm{M}_{\mathrm{P} 2}$, and $\mathrm{M}_{\mathrm{P} 3}$ are the readings of the scale at Support Point-1, Support Point-2, and Support Point-3, respectively. The horizontal positions $\mathrm{P}_{\mathrm{P} 1}, \mathrm{P}_{\mathrm{P} 2}$, and $\mathrm{P}_{\mathrm{P} 3}$; the center of rotation CoR $(\mathrm{O})$; and the angle of rotation $\theta$ are manually measured using the pictures taken by the digital network cameras [image capture resolution: $640 \times 480$, model: BL-C1, manufacturer: Panasonic Co., Ltd. (Japan)] placed at a distance of approximately 2.4 meters above the board. The rotation angle is manually measured using the printed overlapped images that are taken by the fixed digital camera with the help of a straightedge and protractor. Here, we did not compensate the distortion of the image due to the 
perspective projection of the camera when we estimate the center of rotation. The distortion is thought to be negligible because the camera is placed sufficiently far from the board (approximately $2.4 \mathrm{~m}$, compared to the body thickness of less than $0.2 \mathrm{~m}$ and lens center line to margin length of about $0.5 \mathrm{~m}$ ).

Figure 2. Setup for measuring the center of mass CoM of a subject body.

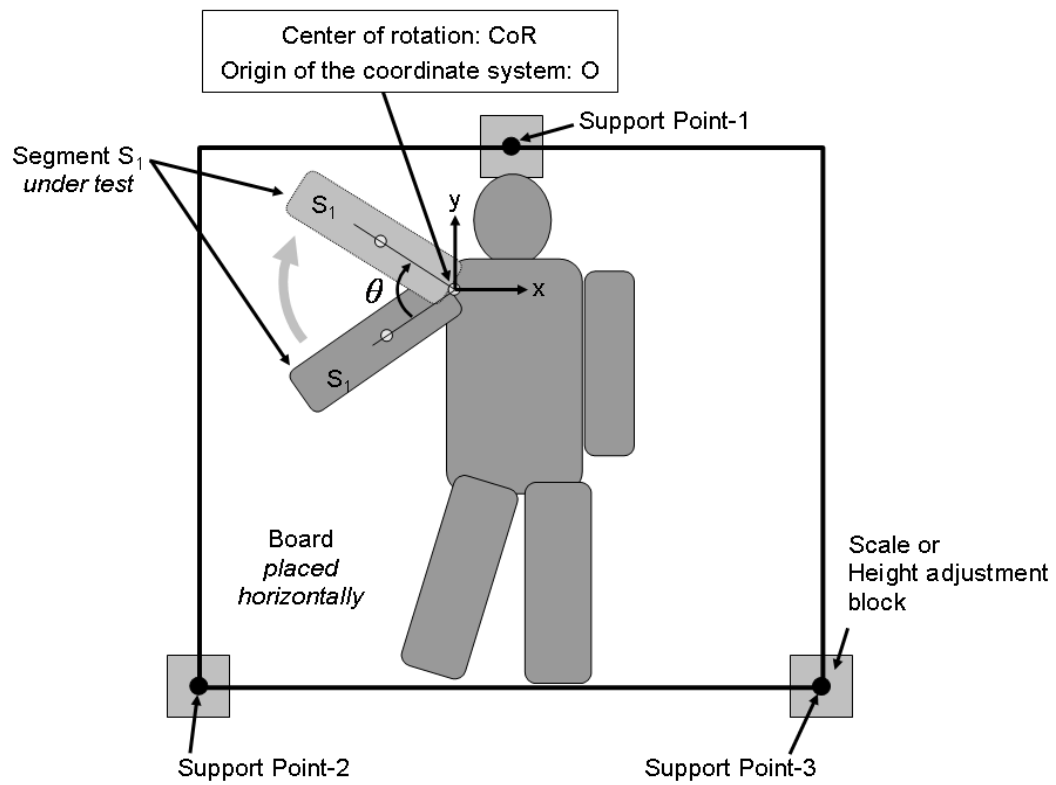

\section{Experiment with a Dummy Mass}

\subsection{Experiments}

The first-order mass moment $\mathrm{M}^{(1)}$ of a dummy mass is evaluated for demonstrating the performance of the proposed method. Figure 3 shows the overlapped pictures, which correspond to a set of measurements.

Figure 3. Overlapped pictures used in the analysis (subject: dummy mass, $\mathrm{M}_{1}: 3.013 \mathrm{~kg}$, $\left.\mathrm{M}^{(1)}{ }_{1}: 116.3 \mathrm{~kg} \cdot \mathrm{cm}\right)$.

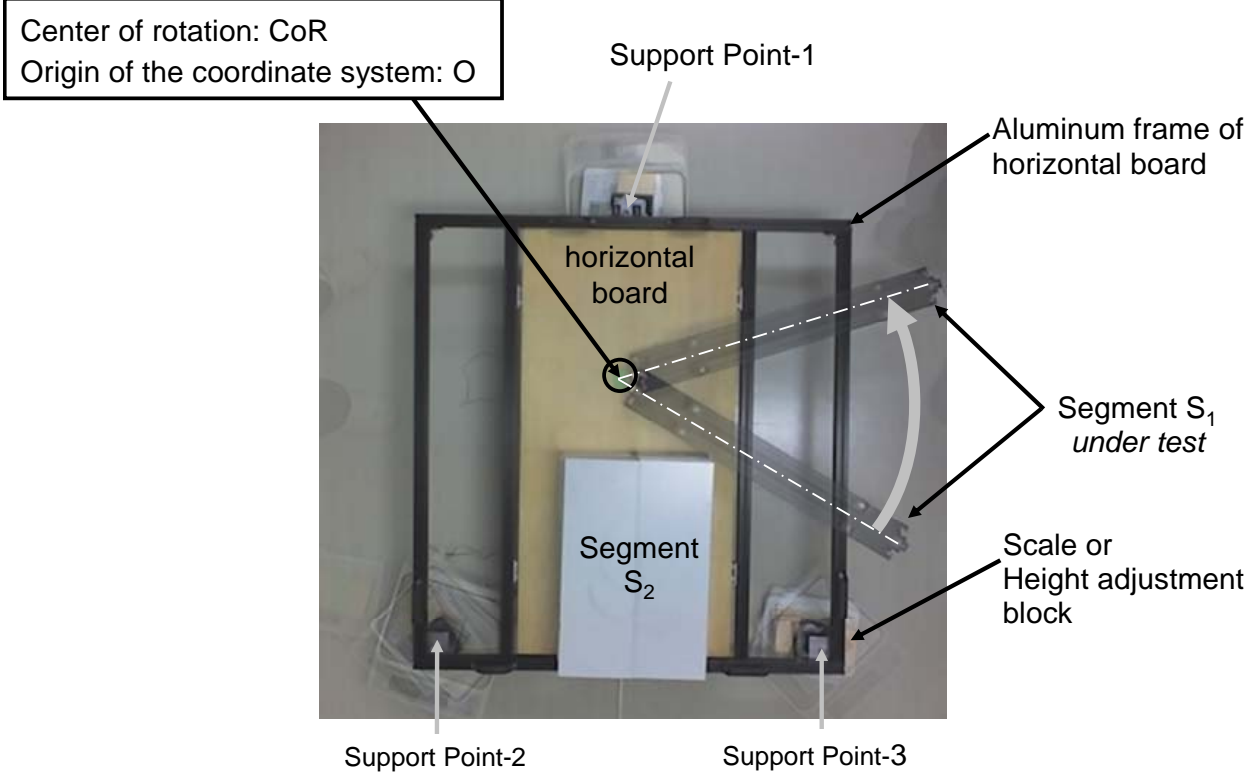


An aluminum bar (mass: $3.013 \mathrm{~kg}$, length: $77.2 \mathrm{~cm}$ ) is used as the segment under test $\mathrm{S}_{1}$, which simulates an arm or a leg under the test. The other aluminum block with a mass of $52.066 \mathrm{~kg}$ is also used as segment $S_{2}$, which simulates the rest of the human body. Both $\mathrm{S} 1$ and $\mathrm{S} 2$ are placed on the horizontal board with a mass of $11.627 \mathrm{~kg}$. The center of rotation CoR and the origin of the coordinate system $\mathrm{O}$ are defined at the end of segment $\mathrm{S}_{1}$. The first-order mass moment $\mathrm{M}^{(1)}$ of segment $\mathrm{S}_{1}$ around the origin $\mathrm{O}$ (the center of rotation $\mathrm{CoR}$ ) is calculated to be $116.3 \mathrm{~kg} \cdot \mathrm{cm}$. In the experiment, 30 sets of measurements are conducted with a rotation angle between $30^{\circ}$ and $180^{\circ}$.

\subsection{Results}

Figure 4 shows the relation between the 30 measured values of $\mathrm{M}^{(1)}$, their mean value $\mathrm{M}^{(1)}$ mean, and the calibrated value $\mathrm{M}^{(1)}$ cal versus the rotation angle. The mean value $\mathrm{M}^{(1)}$ mean and the calibrated value $\mathrm{M}^{(1)}$ cal are $117.6 \mathrm{~kg} \cdot \mathrm{cm}$ and $116.3 \mathrm{~kg} \cdot \mathrm{cm}$, respectively. The standard deviation of the measured values of $\mathrm{M}^{(1)}$ is $1.619 \mathrm{~kg} \cdot \mathrm{cm}$, which corresponds to $1.4 \%$ of the calibrated value $\mathrm{M}^{(1)}$ cal. The root mean square value (RMS value) of the difference between the measured values of $\mathrm{M}^{(1)}$ and the calibrated value $\mathrm{M}^{(1)}$ cal is $2.0 \mathrm{~kg} \cdot \mathrm{cm}$, which corresponds to $1.7 \%$ of the calibrated value $\mathrm{M}^{(1)}$ cal. This value is considered to be the relative standard uncertainty of a single set of measurements of $\mathrm{M}^{(1)}$.

Figure 4. Relationship between the 30 measured values of $\mathrm{M}^{(1)}$, their mean value $\mathrm{M}^{(1)}$ mean, and the calibrated value $\mathrm{M}^{(1)}$ cal versus the rotation angle $\theta$.

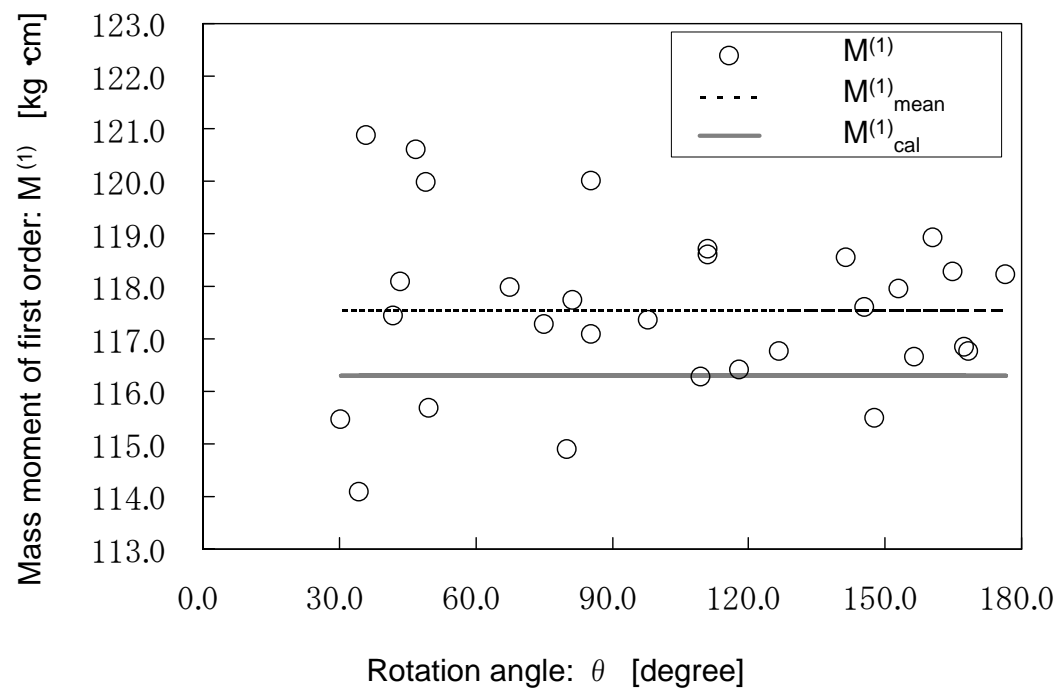

\section{Experiments with a Human Body}

\subsection{Experiments}

The first-order mass moment $\mathrm{M}^{(1)}$ of a leg of a human body is measured using the proposed method. Figure 5 shows the overlapped pictures, which correspond to a set of measurements. In the experiments, 15 sets of measurements are conducted with a constant rotation angle of $48.8^{\circ}$. The total mass of the subject human body, including the clothes, and that of the frame are $63.460 \mathrm{~kg}$ and $15.569 \mathrm{~kg}$, respectively. The rotation center $\mathrm{RoC}$ is estimated from the overlapped pictures such as Figure 5. 
Figure 5. Overlapped pictures used in the analysis.

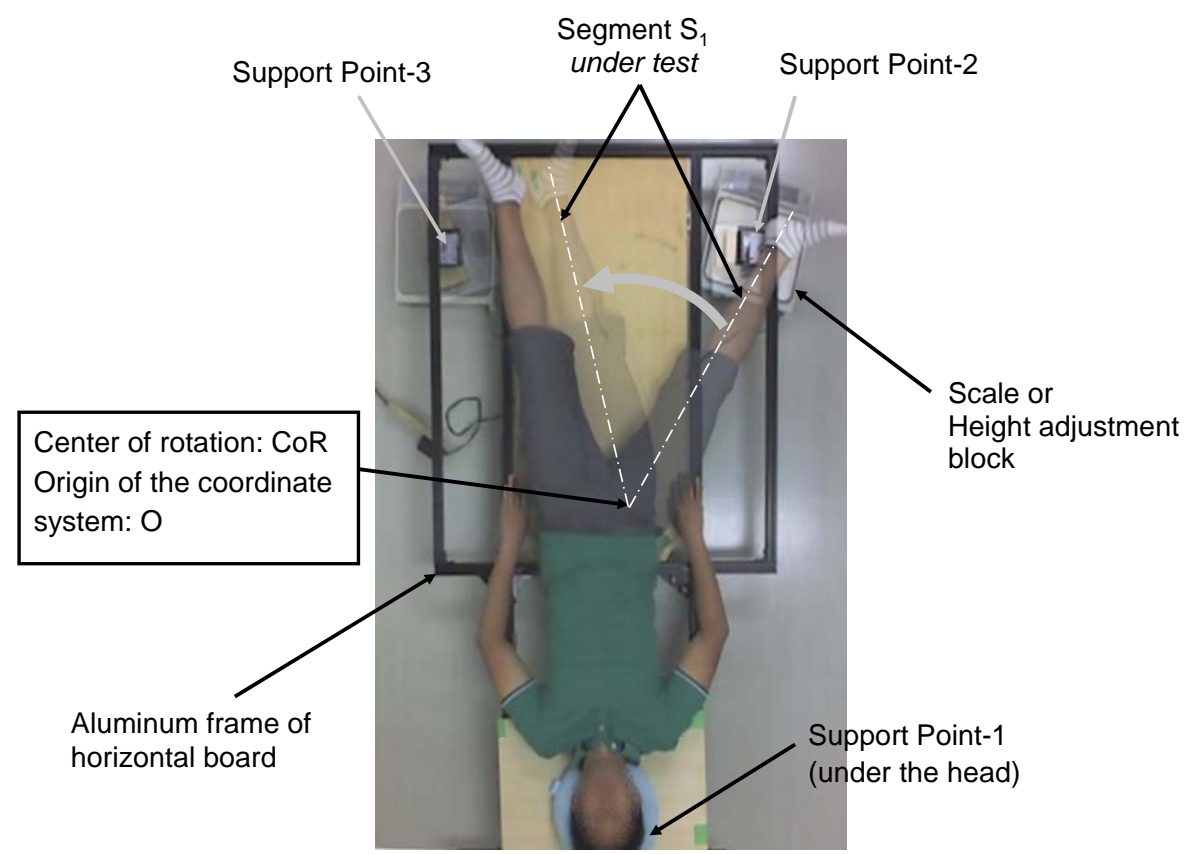

\subsection{Results}

Figure 6 shows the relationship between the 15 measured values of $\mathrm{M}^{(1)}$ and their mean value $\mathrm{M}^{(1)}$ mean versus the order of the set of the measurement. The mean value of the 15 measured values of $\mathrm{M}^{(1)}$ is $388.8 \mathrm{~kg} \cdot \mathrm{cm}$. The standard deviation of the 15 measured values of $\mathrm{M}^{(1)}$ is $5.3 \mathrm{~kg} \cdot \mathrm{cm}$, which corresponds to $1.4 \%$ of the mean value.

Figure 6. Relationship between the 15 measured values of $\mathrm{M}^{(1)}$ and their mean value $\mathrm{M}^{(1)}$ mean versus the order of the set of measurements $i$.

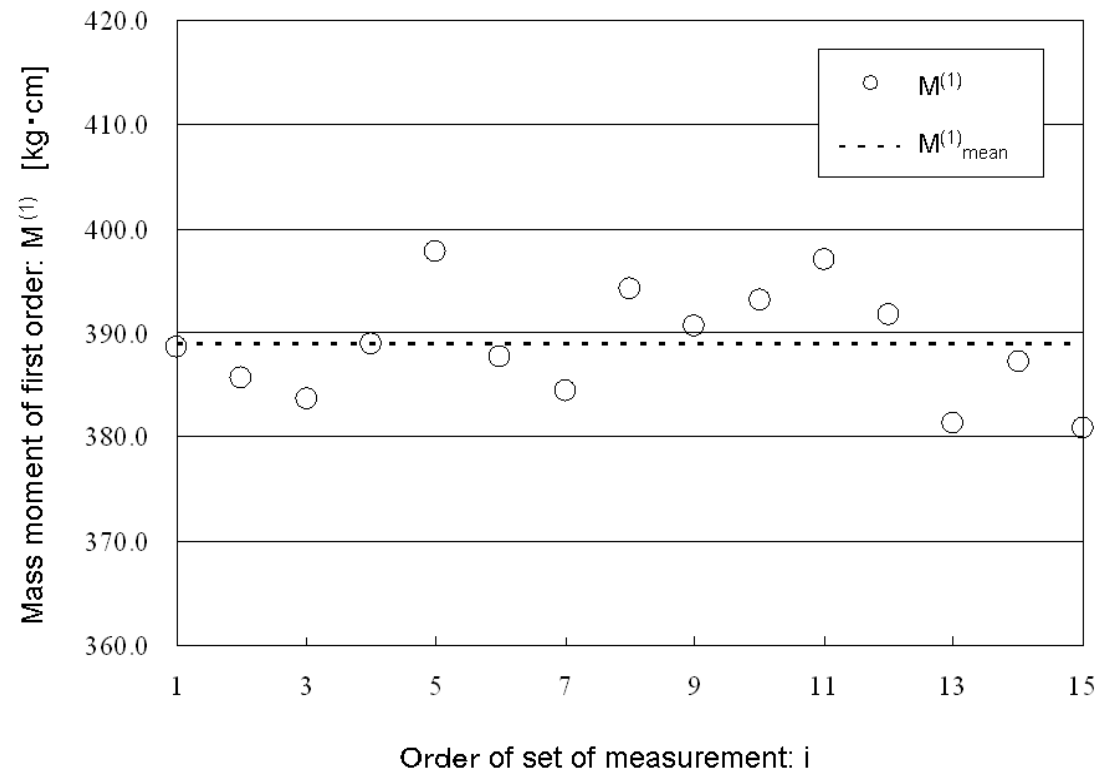




\section{Discussion}

The relative standard uncertainty of a single set of measurements of $\mathrm{M}^{(1)}$ in the dummy mass experiment is estimated to be $1.7 \%$. The standard deviations of the values of $\mathrm{M}^{(1)}$ in the experiments using the dummy mass for 30 measurements with various rotation angles and the human body for 15 measurements with a constant rotation angle are both $1.4 \%$ of the mean value. Although these error values are not directly comparable, the random errors of both the experiments are thought to be at the same level. This indicates that the change of posture of the other part of the human body during one set of measurements can be ignored even when the leg is rotated. With regard to the bias error, the bias error in the human body experiment might be slightly larger than that in the dummy mass experiment since the accuracy of the estimation of the rotation center CoR in the human body experiment might be lesser than that in the dummy mass experiment. In the experiments, the rotation canter CoR and the rotation angle $\theta$ are manually measured using the printed overlapped images that are taken by the fixed digital camera with the help of a straightedge and protractor. The accuracy of estimating the rotation center will be improved with more careful consideration $[12,13]$, such as considering 3-dimensional anatomical structure.

The measured value will be useful as a reference for evaluating the uncertainty of the body segment inertial parameters (BSPs) estimated using an indirect method, in which the mass and the center of mass of a segment are estimated [5,6]. In the evaluation, the value of the first-order mass moment $\mathrm{M}^{(1)}$ of a body segment, which is directly measured using the proposed method, is compared with the value, which is calculated from the mass, the center of mass and the center of rotation estimated using the indirect method.

In the experiment, only one scale is used for measuring the weights applied at the three supporting points of the board. Therefore, during the measurements of the three points, the subject should not be moved; this requires a certain amount of effort in the case of the human subject. If three scales are used, then the weights at the three points can be obtained immediately and the measurement time can be reduced.

\section{Conclusions}

A simple and direct method for measuring the first-order mass moment $\mathrm{M}^{(1)}$ of the human body segment has been proposed. By using the proposed method, the first-order mass moment of the body segment can be directly measured by using only one precision scale and one digital camera. The relative standard uncertainty of a single set of measurements of $\mathrm{M}^{(1)}$ in the dummy mass experiment is estimated to be $1.7 \%$. The measured value will be useful as a reference for evaluating the uncertainty of the body segment inertial parameters (BSPs) estimated using an indirect method.

\section{Acknowledgements}

This work was supported in part by a research-aid fund of the Asahi Glass Foundation, and Grant-in-Aid for Scientific Research (B) 21360196(KAKENHI 21360196). 


\section{References}

1. Durkin, J.L.; Dowling, J.J.; Andrews, D.M. The measurement of body segment inertia parameters using dual energy X-ray absorptiometry. J. Biomech. 2002, 35, 1575-1580.

2. Durkin, J.L.; Dowling, J.J.; Scholtes, L. using mass distribution information to model the human thigh for body segment parameter estimation. J. Biomech. Eng. 2005, 127, 455-464.

3. Durkin, J L.; Dowling, J.J. Body segment parameter estimation of the human lower leg using an elliptical model with validation from DEXA ann. Biomed. Eng. 2006, 34, 1483-1493.

4. Lee, M.K.; Le, N.S.; Fang, A.C.; Koh, M.T. Measurement of body segment parameters using dual energy X-ray absorptiometry and three-dimensional geometry: An application in gait analysis. $J$. Biomech. 2009, 42, 217-222.

5. Davidson, P.L.; Wilson, S.J.; Wilson, B.D.; Chalmers, D.J. Estimating subject-specific body segment parameters using a 3-dimensional modeller program. J. Biomech. 2008, 41, 3506-3510.

6. Wicke, J.; Dumas, G.A.; Costigan, P.A. A comparison between a new model and current models for estimating trunk segment inertial parameters. J. Biomech. 2009, 42, 55-60.

7. Fujii, Y.; Shimada, K. Instrument for measuring the mass of an astronaut. Meas. Sci. Technol. 2006, 17, 2705-2710.

8. Fujii, Y.; Shimada, K.; Maru, K.; Yokota, M.; Hashimoto, S.; Nagai, N.; Sugita, Y. Instrument for measuring the body mass of astronaut. Trans. Space Sci. Jpn. 2009, 7, Th_1-Th_6.

9. Fujii, Y.; Shimada, K.; Maru, K. Instrument for measuring the body mass of astronauts under microgravity conditions. Microgravity Sci. Technol. 2010, 22, 115-121.

10. Barbier, F.; Allard, P.; Guelton, K.; Colobert, B.; Godillon-Maquinghen, A.-P. Estimation of the 3-D center of mass excursion from force-plate data during standing. IEEE Trans. Rehab. Eng. 2003, 11, 31-37.

11. McKinon, W.; Hartford, C.; Zio, L.; van Schalkwyk, J.; Veliotes, D.; Hofmeyr, A.; Rogers, G. The agreement between reaction-board measurements and kinematic estimation of adult male human whole body centre of mass location during running. Physiol. Meas. 2004, 25, 1339-1359.

12. Veeger, H.E.J. The position of the rotation center of the glenohumeral joint. J. Biomech. 2000, 33, 1711-1715.

13. Koo, S.; Andriacchi, T.P. The knee joint center of rotation is predominantly on the lateral side during nomal walking. J. Biomech. 2008, 41, 1269-1273.

(C) 2010 by the authors; licensee MDPI, Basel, Switzerland. This article is an open access article distributed under the terms and conditions of the Creative Commons Attribution license (http://creativecommons.org/licenses/by/3.0/). 